\title{
Implicaciones del endotelio en la insuficiencia cardiaca
}

\author{
Implications of endothelium dysfunction in heart failure
}

\author{
Beatriz Wills Sanín ${ }^{1}$, Andrés Felipe Buitrago ${ }^{2,3}$ \\ 1 Médica Rural de Cuidado Crítico Fundación Santa Fe de Bogotá, Colombia. \\ ${ }^{2}$ Especialista en Medicina Interna, Cardiología y Epidemiologia, Hospital Universitario Fundación Santa Fe de Bogotá, Colombia. \\ ${ }^{3}$ Jefe Asociado del Departamento de Medicina Crítica y Cuidado Intensivo, Hospital Universitario Fundación Santa Fe de Bogotá, Colombia.
}

\begin{abstract}
Resumen
El papel del endotelio en la insuficiencia cardiaca (IC) ha sido el centro de intensa investigación, puesto que tiene importantes implicaciones sistémicas expresadas en su efecto vasomotor, hemostático e inflamatorio. Existen diferencias individuales en el patrón de la disfunción endotelial de acuerdo a la etiología y severidad de la IC. La disfunción endotelial tiene un importante valor pronóstico en la IC y, aunque aún necesitamos conocer más aspectos de relevancia clínica, es evidente que esta condición se relaciona con desenlaces negativos en estos pacientes. Estamos a la espera de nuevos agentes farmacéuticos dirigidos especificamente al endotelio vascular. En esta revisión, nuestro objetivo es ofrecer un análisis crítico y contemporáneo de la evidencia disponible sobre las consecuencias patológicas y pronóstico de las anomalias endoteliales que definen el inicio, la historia natural y, en última instancia, los desenlaces en la IC.
\end{abstract}

Palabras clave: Endotelio, implicaciones en falla cardiaca, óxido nítrico, terapia.

Abstract

The role of the endothelium in heart failure has been the focus of intense research, since it has important biologic implications. There are individual differences in the pattern of endothelial dysfunction according to the etiology and severity of heart failure. Endothelial dysfunction is also associated with negative outcomes. We are eagerly waiting for new pharmacological agents targeted specifically to the vascular endothelium. In this review, we aim to provide a critical analysis of the available evidence on the pathological consequences and prognosis of endothelial abnormalities as related to the onset and natural history of heart failure.

Keywords: Endothelium, heart failure, nitric oxide, new agents.

An Fac med. 2014;75(4):353-9 / doi: http://dx.doi.org/10.15381/anales.v75i4.10857

\section{INTRODUCCIÓN}

La insuficiencia cardiaca (IC) afecta la función y estructura cardiaca y esta puede manifestarse como disfunción sisto-diastólica del ventrículo izquierdo o ventrículo derecho, valvulopatía, hipertensión pulmonar y/o arritmias cardiacas. Se ha demostrado que la disfunción endotelial tiene un papel fundamental en el desarrollo de estas alteraciones mencionadas. El endotelio regula el tono vasomotor, la hemostasia, los niveles de antioxidantes y anti-inflamatorios sistémicos que en conjunto determinan el desarrollo de múltiples condiciones cardiovasculares, incluyendo la IC.

El desenlace de los pacientes con IC aguda continúa siendo pobre, con altas tasas de mortalidad y de hospitalización ${ }^{(1)}$. Esto ha impulsado el crecimiento de distintos campos de investigación de la IC, que tiene como objetivo profundizar el conocimiento fisiopatológico para crear alternativas de manejo y así disminuir la carga de la enfermedad en el sistema de salud. En este sentido, el endotelio ha ido adquiriendo un rol central en la fisiopatología de la IC.

\section{METODOLOGÍA}

Inicialmente se planteó el tema de la revisión de literatura teniendo en cuenta que ha a tenido un importante alcance en distintos campos de la cardiología. Posteriormente, se realizó una búsqueda de la literatura en las bases de datos Medline $\boldsymbol{y}$ Pubmed utilizando las palabras clave endotelio, implicaciones en falla cardiaca, óxido nítrico, terapia (las mismas traducidas al inglés). Se seleccionó 29 artículos que permitieron la construcción de este manuscrito.

\section{ENDOTELIO COMO ÓRGANO ENDOCRINO}

El endotelio es la capa celular que recubre la superficie luminal de los vasos sanguíneos. Hasta hace poco, el endotelio fue considerado como una zona inerte sin ningún rol importante. Hoy en día, sabemos que el endotelio tiene funciones esenciales, además de proporcionar el revestimiento de las paredes vasculares. Por ejemplo, el endotelio está involucrado en el equilibrio entre la trombosis y la trombolisis, en la interacción y reclutamiento de las plaquetas, en la función inmune a través de la adhesión de leucocitos, en la regulación del tono vascular, en el control del volumen de fluidos y concentración de electrolitos, en el transporte de hormonas y en la angiogénesis (2).

Las funciones complejas de este gran órgano endocrino permiten conocer la 
situación molecular que ocurre en estas células vasculares que limitan el sistema circulatorio, desde el corazón hasta los capilares más pequeños. Los investigadores en biología vascular han demostrado que el endotelio tiene una amplia influencia en la homeostasis, con la capacidad de actuar como órgano sensorial y efector. A su vez, el endotelio está regulado por distintos receptores asociados a la membrana plasmática, proteínas, partículas transportadoras de lípidos (clatrina), metabolitos, hormonas y proteínas de unión que regulan la interacción a la matriz celular ${ }^{(3)}$.

Las células endoteliales (CE) también son fundamentales en la regulación del flujo sanguíneo. Esto se debe a que, en estado quiescente, estas son capaces de generar una superficie antitrombótica que facilite el flujo del plasma y componentes celulares a lo largo de la circulación. Las alteraciones que ocurren debido al estado inflamatorio y al estrés hemodinámico secundario a la IC interrumpen las actividades homeostáticas e inducen la formación de un microambiente protrombótico y antifibrinolítico. El flujo de sangre también está regulado, en parte por la secreción y absorción de las sustancias vasoactivas del endotelio que actúan de una manera paracrina al contraer $o$ dilatar los lechos vasculares en respuesta a estímulos como la endotoxina ${ }^{(4)}$.

El endotelio también es esencial en la regulación de la coagulación. Las CE controlan el sistema de coagulación mediante la regulación de los sitios de unión de los factores de coagulación a la superficie endotelial. En reposo, las CE mantienen el flujo sanguíneo promoviendo la actividad de sustancias anticoagulantes, al favorecer la acción de las proteínas $\mathrm{C}$ y $\mathrm{S}^{(5)}$.

\section{FISIOPATOLOGÍA DE LA DISFUNCIÓN ENDOTELIAL}

La disfunción endotelial se caracteriza por vasoconstricción, inflamación y un estado protrombótico. La injuria endotelial altera el equilibrio de las propiedades de estas células al favorecer la formación de coágulos a través de la inducción coordinada de moléculas pro-coagulantes y la supresión de mecanismos anticoagulantes. Esto puede ser provocado por la presencia de citoquinas como el factor de necrosis tumoral (TNF, por sus siglas en inglés), aumentado en pacientes con IC, que suprime la formación de trombomodulina, un cofactor anticoagulante. El TNF también induce la expresión del factor tisular, un cofactor pro-coagulante. En conjunto, estos cambios permiten la formación de fibrina en un ambiente pro-inflamatorio, característico de la IC ${ }^{(6)}$.

Los radicales libres también alteran el equilibrio endotelial al disminuir la producción de óxido nítrico $(\mathrm{ON})$, lesionando el endotelio y aumentando la permeabilidad celular. Esto permite que distintas toxinas ingresen al tejido cardiovascular. En la mayoría de los casos, el organismo cuenta con un suministro adecuado de antioxidantes obtenidos de distintas fuentes alimentarias que neutralizan los radicales libres; sin embargo, cuando estos antioxidantes se agotan y hay otros factores coexistentes como la obesidad, el tabaquismo, la diabetes, la hipertensión arterial o incluso el sueño insuficiente, se inicia el proceso de injuria endotelial y con ella la cascada de alteraciones hemodinámicas que termina finalmente en IC ${ }^{(7)}$.

Estas alteraciones están presentes en la mayoría de enfermedades cardiovasculares, como la hipertensión, la enfermedad coronaria, la enfermedad vascular periférica, la diabetes y el enfoque de esta revisión: la IC.

\section{EVIDENCIA DE LA DISFUNCIÓN ENDOTELIAL EN IC}

La investigación en este campo ha sugerido distintas anormalidades en la función endotelial en los pacientes con IC (figura 1). Generalmente esta se evalúa a través del flujo de la arteria braquial en respuesta a distintas moléculas como la acetilcolina. Sin embargo, aún faltan estudios que demuestren con precisión la correlación entre la disfunción endotelial, los parámetros clínicos, la severidad de la IC, la contractilidad cardíaca o el aumento de la presión de oclusión pulmonar.

Existen diferencias en el patrón de la disfunción endotelial de acuerdo a la etiología y la gravedad de la IC. En el caso de IC isquémica, la disfunción endotelial sistémica es esencialmente parte de la misma condición que afecta tanto a arterias como venas, vasos de conductancia y lechos vasculares coronarios, pulmonares y periféricos. Múltiples factores clásicos de la IC isquémica, como la aterosclerosis y la diabetes, contribuyen per se y significativamente al deterioro endotelial. Además, los pacientes con IC isquémica tienen niveles aumentados de proteína $\mathrm{C}$ reactiva y de superóxido dependiente de nicotinamida adenina dinucleótido fosfato (NADPH), contribuyendo a la injuria endotelial ${ }^{(8)}$.

El patrón de la disfunción endotelial es más heterogéneo en la IC no isquémica. En estos casos hay menor compromiso sistémico. De hecho, estos sujetos frecuentemente tienen un endotelio funcional conservado en las arterias periféricas, pues en ellos la disfunción endotelial puede manifestarse únicamente en los vasos coronarios. En efecto, los expertos han demostrado que sí hay un deterioro significativo en el endotelio coronario en los pacientes con disfunción del ventrículo izquierdo independiente de la etiología de la IC. Incluso el endotelio está comprometido en la mayoría de los pacientes con miocardiopatía dilatada idiopática, a pesar de que en estos casos las arterias coronarias pericárdicas parezcan normales. El compromiso coronario ocasiona, por supuesto, una disminución en el flujo sanguíneo al miocardio y una reducción significativa en la capacidad de aumentar la liberación de ON. Esta disfunción endotelial está asociada a la alteración estructural, a cambios de la geometría cardiaca y a un aumento en los niveles de péptido natriurético cerebral (BNP) ${ }^{(9)}$. 


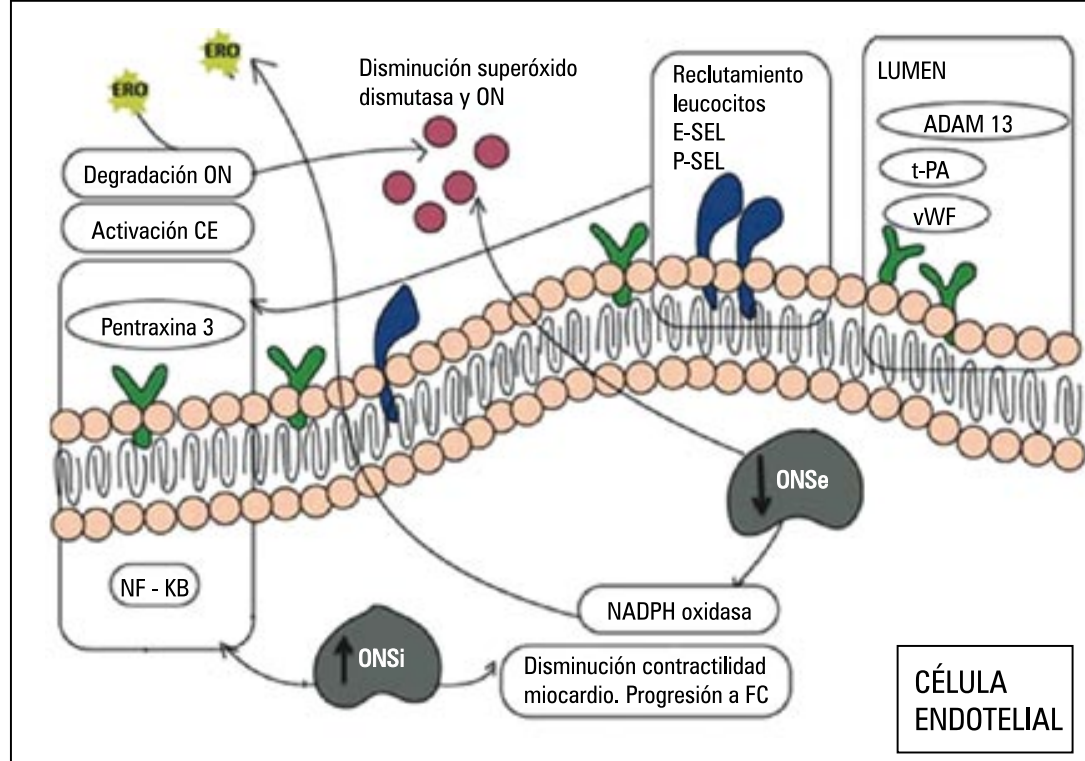

CE: célula endotelial; E-sel, E-selectina soluble; ONSe, óxido nítrico sintasa endotelial; ONSi, óxido nítrico sintasa inducible; NFkB nuclear factor KB; ON, óxido nítrico; P-sel, P-selectina; ERO, especies reactivas de oxígeno. Modificado de Shantsila $E_{1}$ et al . Endothelial function in heart failure ${ }^{\mid 6 !}$.

Figura 1. Anormalidades del endotelio relacionados con falla cardiaca (FC). La disfunción endotelial en la FC se acompaña de estrés oxidativo, contribuyendo a la degradación acelerada de ON. El endotelio activado produce distintas moléculas proinflamatorias, como la pentraxina. También atrae y recluta leucocitos en la pared vascular. La injuria endotelial además contribuye a un estado protrombótico asociado con aumento en la producción del factor de von Willebrand (VWF), factor tisular y t-PA (tissue-plasminogen activator). En esta situación, hay reducción de la expresión de ADAMTS 13.

Es importante estudiar las CE de diversos órganos periféricos. Sin embargo, al tratarse de insuficiencia cardiaca congestiva (ICC), debemos considerar sobre todo las CE cardiacas, incluyendo las CE de los vasos coronarios, de los capilares intramiocárdicos y del endocardio, donde las CE están directamente en comunicación con los cardiomiocitos. Específicamente, este deterioro endotelial coronario tiene un papel fundamental en la patogénesis de la IC, afectando tanto la resistencia microvascular como la conductancia de los grandes vasos, que se desarrolla en paralelo al deterioro de la contractilidad cardiaca ${ }^{(10)}$.

La contribución del endotelio cardiaco en conjunto con el endotelio vascular pulmonar es fundamental, porque representa la mayor superficie endotelial corporal y es fuente esencial de distintos mediadores endoteliales. De hecho, en ambientes experimenta- les se ha comprobado que la disfunción endotelial contribuye significativamente al desarrollo de la insuficiencia cardíaca en tejido isquémico cardiaco (figura 2). Además de afectar el estado hemodinámico cardíaco, hay una estrecha relación entre las CE con los cardiomiocitos y los fibroblastos. Juntos orquestan la remodelación cardiaca, la fibrosis y la angiogénesis, reflejando la naturaleza compleja de la IC ${ }^{(9)}$.

Además de influir los vasos coronarios, también hay disfunción endotelial generalizada que compromete tanto la circulación sistémica como la pulmonar en los pacientes con IC sistólica. Se ha demostrado que el ON exhalado está aumentado en los pacientes con cor pulmonale, indicando la importancia del rol del ON como agente contraregulatorio en la disminución del flujo sanguíneo propio de la IC. En el cor pulmonale hay una reducción de la liberación del ON durante el ejercicio, que junto con la hipoxia contribuyen al daño endotelial ${ }^{(11)}$.

Por otra parte, la rigidez ventricular y vascular de la circulación sistémica y pulmonar también desempeña un rol en la fisiopatología de la IC con fracción de eyección preservada (ICFEP). La disfunción endotelial detectada en individuos sanos con presión arterial braquial normal está asociada con aumento de la presión venosa central y con rigidez arterial. Esto sugiere que el compromiso endotelial sistémico puede ser un fenómeno primario en el desarrollo de la hipertensión arterial sistémica que produce aumento en el estrés de la pared del ventrículo izquierdo, resultando en hipertrofia y disfunción diastólica en ICFEP ${ }^{(12)}$.

En la circulación pulmonar, la disfunción endotelial también es un evento temprano que conduce a hipertensión pulmonar en el contexto de IC. Los cambios que ocurren secundarios a la disfunción endotelial en la circulación sistémica como pulmonar tienen características distintas. En primer lugar, la circulación pulmonar es controlada por flujo, mientras que la circulación sistémica es regulada por presión y, por lo tanto, podría ser más susceptible a la influencia de la tensión de cizallamiento y a la disfunción endotelial ${ }^{(11)}$.

El deterioro de las propiedades elásticas arteriales también afecta la disfunción vascular sistémica de la IC. Estos cambios parecen ser secundarios a la remodelación arterial que ocasiona hipertrofia vascular observada en los pacientes con IC crónica (ICC) severa. En efecto, la hipertrofia vascular se correlaciona con disminución en la respuesta arterial a los nitratos ${ }^{(13)}$.

La naturaleza sistémica de la disfunción endotelial observada en pacientes con IC es coherente con la percepción que le hemos atribuido al endotelio como un órgano independiente. Sin embargo, este enfoque también reconoce la diversidad del fenotipo y las distintas funciones de las CE localizadas dentro de los diferentes segmentos del árbol vascular. 


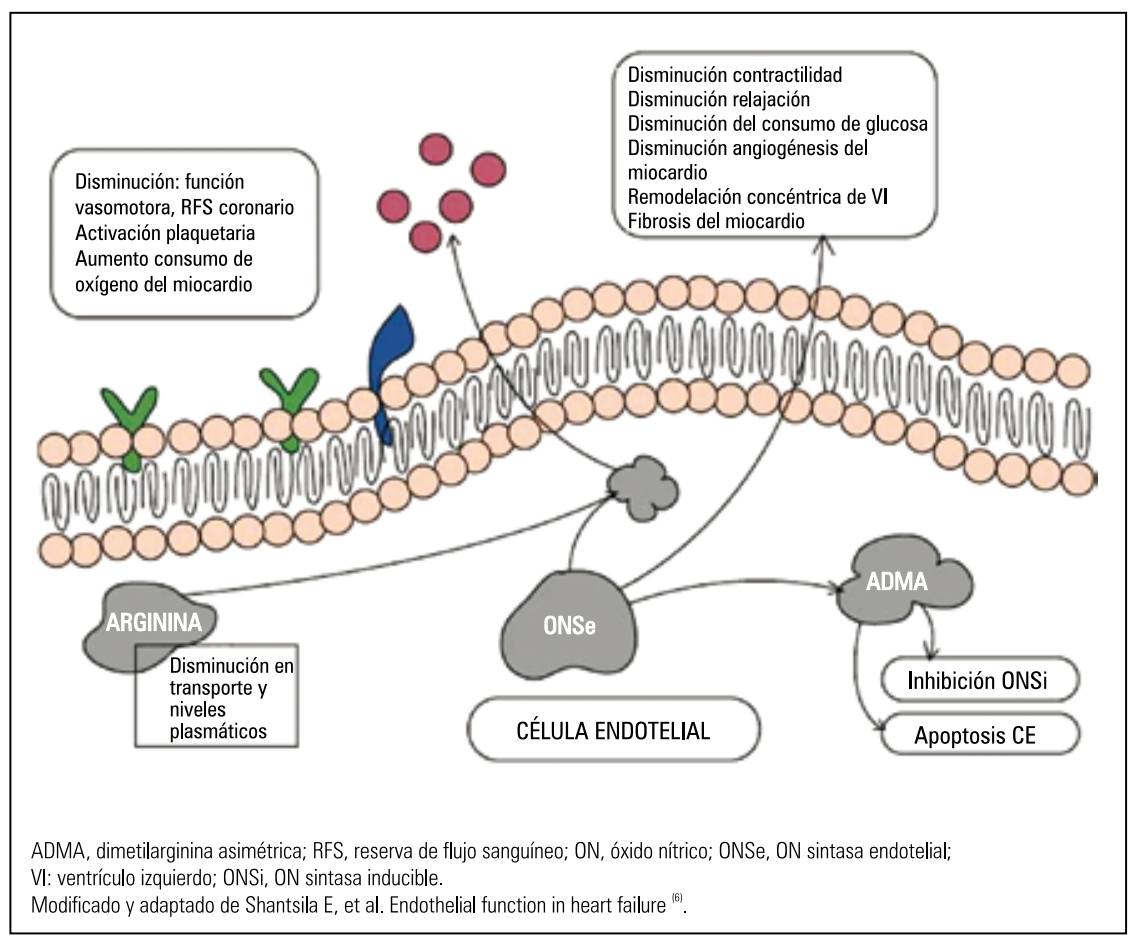

Figura 2. Mecanismos y efectos de la función endotelial vasomotora en la insuficiencia cardiaca. La disfunción endotelial vasomotora es causada por la disminución de la expresión de eNOS y la producción de NO; la disponibilidad de arginina también está disminuida. Además, hay un aumento del inhibidor de eNOS endógeno: el ADMA. En la insuficiencia cardíaca, estas anormalidades reducen el flujo sanguíneo coronario de reserva, llevan a un aumento del consumo miocárdico de oxígeno, al deterioro de la función cardiaca, de la angiogénesis y a remodelación cardiaca que, en últimas, resulta en fibrosis irreversible.

\section{IMPLICACIÓN CLÍNICA DE LA IC DE LA DISFUNCIÓN ENDOTELIAL}

La relevancia clínica de la disfunción endotelial en los pacientes con IC deriva de estudios prospectivos que muestran que la disfunción endotelial puede predecir de manera independiente desenlaces cardiovasculares negativos como: disminución de la clase funcional y de la fracción de eyección del ventrículo izquierdo (FEVI), mortalidad cardiovascular, infarto agudo de miocardio y reingreso hospitalario por descompensación de IC ${ }^{(14)}$.

La disfunción endotelial tiene un importante valor pronóstico en la IC, pero su aplicación clínica ha sido obstaculizada por las limitaciones metodológicas de su evaluación. El compromiso endotelial en IC isquémica está asociado con disminución en la perfusión de reserva miocárdica evaluada por tomografía por emisión de positrones. De manera semejante, la respuesta arterial a la acetilcolina y el flujo sanguíneo vascular se relacionan significativamente con eventos desfavorables de la IC independiente de su etiología ${ }^{(15)}$. La disfunción endotelial pericárdica es también un predictor independiente de eventos cardiovasculares y muerte prematura en pacientes con trasplante cardiaco ${ }^{(16)}$.

Un estudio observacional de 321 pacientes en Japón conducido por Akiyama y col ${ }^{(14)}$ destacó la asociación significativa entre la disfunción endotelial y el desenlace clínico a los 20 meses de seguimiento en pacientes con ICFEP. En este estudio, la función endotelial fue evaluada mediante la reactividad hiperémica por tonometría arterial periférica. La disfunción endotelial -definida por un índice de hiperemia reactiva por debajo de 0,49- se relacionó de forma independiente con ICFEP, al igual que el aumento del índice de masa corporal y con menor tasa de filtración glomerular. Los autores también demostraron que los niveles de la proteína $C$ reactiva (PCR) estaban aumentados en los pacientes con IC, sugiriendo que este es un estado esencialmente pro-inflamatorio.

\section{MARCADORES DE DISFUNCIÓN ENDOTELIAL EN INSUFICIENCIA CARDIACA}

Algunos estudios han comprobado aumento de los marcadores plasmáticos de activación (como la E-selectina) y daño endotelial (como el factor de von Willebrand, fvW) en la IC. Sin embargo, los expertos sugieren que distintas comorbilidades como la diabetes, frecuentes en los pacientes con ICC, son un factor de confusión. Otra evidencia de daño endotelial, son precisamente las CE circulantes (CEC), que se encuentran aumentadas en la IC aguda y crónica ${ }^{(16)}$.

La endotelina-1 es producida por el endotelio y por las células musculares lisas vasculares; es conocida como la molécula más potente que ocasiona vasoconstricción. La sobreexpresión de esta también refleja la disfunción endotelial. Como es de esperarse, los niveles de endotelina-1 plasmáticos están incrementados en la IC ${ }^{(17)}$.

Si bien los ensayos clínicos del antagonista del receptor de endotelina-1 no han sido alentadores, el uso de betabloqueadores y de estatinas sí ha demostrado reducir los niveles de endotelina-1, posiblemente contribuyendo a los efectos protectores que se observa en los pacientes que reciben estas terapias. Incluso, el ensayo A-heFT indicó que la disfunción endotelial se correlaciona con mortalidad, morbilidad y con el estado funcional de los pacientes afroamericanos con IC ${ }^{(18)}$.

El estrés al que están sometidos los miocitos por la sobrecarga de volumen 
y presión también produce aumento en la expresión génica de los péptidos natriuréticos atrial y cerebral (ANP / $\mathrm{BNP}$ ). La evidencia indica que la evaluación del BNP y del NT-proBNP son una herramienta objetiva para el monitoreo y manejo de pacientes con IC crónica. Un estudio demostró que el flujo sanguíneo cutáneo microvascular medido estaba reducido en los pacientes con ICC. Los autores atribuyen estos resultados a una posible desensibilización de los receptores de ANP y $\mathrm{BNP}$; y a una reducción en los niveles de GMPc que regulan la vasodilatación inducida por $\mathrm{ON}^{(19)}$.

\section{TERAPIAS DIRIGIDAS AL ENDOTELIO EN IC}

Como se mencionó previamente, la disfunción endotelial es el paso inicial en las patogénesis de múltiples enfermedades crónicas, incluyendo la IC. Los avances en la comprensión de la función endotelial han permitido el desarrollo de distintas estrategias terapéuticas que pretenden restaurar el estado de reposo de las CE. Muchos medicamentos, como el sildenafil yLCZ696 (medicamento de acción dual al inhibir la neprilisina y el receptor de angiotensina II) activan directa o indirectamente la vía del GMPc; estos actualmente están siendo estudiados en ensayos clínicos de fase III para el manejo de ICFEP (20).

Otros medicamentos que también pretenden restituir el endotelio incluyen los inhibidores de la enzima convertidora de angiotensina (IECA), las estatinas y los sensibilizadores de insulina. También se han planteado otros agentes dirigidos a la enzima óxido nítrico sintasa endotelial (eNOS), como los folatos o la tetrahidrobiopterina $\left(\mathrm{BH}_{4}\right)$.

Múltiples estudios experimentales han demostrado resultados prometedores con los inhibidores de otras vías de señalización, como rho-quinasa, transcetolasa, geraniltransferasa y activadores de la proteinquinasa $\mathrm{A}$ (PKA) y $\mathrm{B}$
(Akt); estos han sido postulados como diana terapéutico en diferentes enfermedades cardiovasculares; no serían exclusivos para IC.

La activación excesiva del sistema renina angiotensina aldosterona interrumpe la señalización del ON. En consecuencia, todos los estudios disponibles sugieren que el bloqueo de esta vía no solo a través de los IECA, sino también con la administración de los antagonistas de los receptores de mineralocorticoides, mejora la función endotelial y reduce los niveles de fvW en los pacientes con IC. Sin embargo la habilidad de restaurar la función del endotelio varía con diferentes agentes farmacológicos y su dosis (por ejemplo, es necesario una dosis mayor de enalapril para alcanzar este beneficio). Estos efectos en el endotelio han sido atribuidos al aumento de la óxido nítrico sintetasa, a la inhibición de la apoptosis endotelial y a la reducción de los prostanoides vasoconstrictores ${ }^{(21)}$.

Las estatinas tienen propiedades independientes de su efecto hipolipemiante que, como los IECA, también afectan la regulación de la función endotelial. Estos medicamentos ayudan a reducir el estrés oxidativo, a mantener la producción de $\mathrm{ON}$, la vasodilatación y la angiogénesis. Además, se ha encontrado que el manejo con estatinas -a diferencia de otras moléculas como ezetimibe- se acompaña de un aumento de la fracción de eyección del VI (FEVI). Estos efectos también son dependientes del tipo y de la dosis utilizada (22).

La suplementación con arginina también ha llamado la atención en la comunidad científica, siendo este aminoácido el precursor del ON. Un estudio realizado en humanos mostró que una dosis inferior (8 gramos al día) de L-arginina en lugar de 20 gramos al día mejoró la función endotelial, dado por aumento en la distancia recorrida en la caminata de 6 minutos y en la distensibilidad arterial; adicionalmente, se redujeron los niveles de endotelina-1 circulante. Sin embargo, el exceso de L-arginina puede ser perjudicial para el endotelio y para la función hepática ${ }^{(23)}$.

La vitamina $\mathrm{C}$ también ha sido estudiada en este campo, puesto que inhibe la apoptosis de las CE que ocurre en la IC. Sin embargo, dados los resultados infructuosos de ensayos clínicos sobre la suplementación con vitamina C como antioxidante, esta no debe ser recomendada de forma rutinaria en IC ${ }^{(24)}$.

La hormona relaxina regula distintos parámetros hemodinámicos durante el embarazo. Estudios recientes han demostrado que este péptido también tiene efecto como vasodilatador renal y sistémico, en la distensibilidad arterial y en el gasto cardíaco de los pacientes con IC. Los ensayos clínicos han confirmado que la relaxina disminuye la presión pulmonar en cuña y la resistencia vascular sistémica en los pacientes con IC compensada. El estudio de fase III (RELAXin in Acute Heart Failure [RELAX-AHF]) demostró que la infusión de 48 horas de serelaxina (la forma recombinante de relaxina-2 humana) en pacientes con ICA mejora significativamente los desenlaces clínicos, como la disnea, la descompensación clínica de ICA, la mortalidad total y cardiovascular a los 180 días de seguimiento ${ }^{(25)}$.

Los receptores de la relaxina, RXFP1 se expresan en células del músculo liso vascular y endotelial de arterias y venas. Se ha propuesto un mecanismo dependiente del endotelio para explicar las acciones vasodilatadoras de la relaxina en las pequeñas arterias renales. Esto implica un aumento de la actividad vascular de las metaloproteinasas (MMP-2 y MMP-9), del factor de crecimiento endotelial vascular y del factor de crecimiento placentario. Estos mecanismos conducen a la activación de los receptores de la endotelina B y del óxido nítrico $(\mathrm{ON})$. Por otra parte, se ha sugerido que la vasodilatación que ocurre por la relaxina es mediada por la estimulación de fosfatidilinositol-3 -quinasa (PI3K) a la proteína quinasa B (Akt) y a la enzima óxido nítrico sintasa endotelial. Adicionalmente, la 
vasodilatación también puede ser secundaria al aumento de los niveles de prostaciclina desencadenada por la relaxina ${ }^{(26)}$.

Es importante tener en cuenta que la mejoría en la función endotelial no necesariamente se traduce en beneficios clínicos de la IC. Esto puede ser debido a la metodología de los ensayos clínicos que compararon las intervenciones mencionadas, los cuales en su mayoría tuvieron un seguimiento a corto plazo. Además, los efectos de estas terapias como los antioxidantes no se limitan únicamente al endotelio, pues tienen múltiples efectos biológicos en distintos tejidos relacionados con el sistema vascular. Otra posible explicación es precisamente la naturaleza compleja de la IC: intervenir el endotelio y optimizar su función puede ser insuficiente para cambiar el desenlace definitivo de los pacientes con IC. El desarrollo de nuevas terapias dirigidas específicamente al endotelio pueden ofrecer oportunidades adicionales en el pronóstico, en la tasa de hospitalización y mortalidad de los pacientes con IC.

Es reconocido ampliamente que la respuesta neuroendocrina es perjudicial para la IC. Sin embargo, hay otras explicaciones biológicas que permiten comprender con mayor profundidad la biología de esta enfermedad. Después del reconocimiento de la efectividad de medicamentos como los IECA, betabloqueadores y antagonistas de mineralocorticoides, aún no tenemos otros medicamentos innovadores que retarden el proceso de remodelación en IC.

Así pues, el papel de los progenitores de la reparación endotelial y vascular ha sido recientemente estudiado. Se ha encontrado recuperación del tejido miocárdico isquémico con la administración selectiva de factores de crecimiento angiogénicos (como el factor vascular endotelial de crecimiento (VEGF) de acción prolongada. Estamos esperando los resultados de los ensayos clínicos de estas moléculas para determinar si pueden ser incluidas en las guías de manejo de IC ${ }^{(11)}$.
En este sentido, la vía de señalización de los receptores Notch ha recibido un interés muy merecido. El entendimiento de esta familia que codifica receptores transmembrana y ligandos viene sobre todo de la investigación en oncología. Aunque su alcance en cardiología hasta ahora se está definiendo, los avances han demostrado que tiene implicaciones muy importantes desde el desarrollo embriológico hasta la remodelación cardiaca post infarto. Por ejemplo, las mutaciones del regulador de señalización Notch 1 están asociadas con la valvulopatía aórtica ${ }^{(27)}$. Las terapias biológicas con anticuerpos monoclonales dirigidas a los receptores Notch $(1,2$, y 4$)$ expresadas en el endotelio están siendo estudiadas actualmente. Parece que sus efectos benéficos en la prevención de la remodelación de la IC se les atribuye a su influencia en la supervivencia y proliferación de las CE y cardiomiocitos ${ }^{(28)}$.

También tendremos que aclarar la utilidad de otros agentes terapéuticos, como el alopurinol, el etanercept, la hormona del crecimiento, anti-renina, anti-endotelina, en estudios clínicos aleatorizados y doble ciegos.

\section{IMPORTANCIA DEL EJERCICIO EN LA INSUFICIENCIA CARDIACA}

El ejercicio físico es una intervención importante en los pacientes con IC. De acuerdo con una revisión sistemática de ensayos clínicos controlados aleatorizados, el ejercicio podría retrasar el progreso fisiopatológico de esta enfermedad. En la ICC, el ejercicio regular inhibe la estimulación neurohormonal y la producción de citoquinas proinflamatorias; además, reduce los niveles de péptido natriurético, la resistencia vascular arterial y atenúa el estrés oxidativo. Estos efectos moleculares se traducen en mejoría de los síntomas y en la calidad de vida de los pacientes con IC ${ }^{(29)}$.

La fuerza de cizallamiento generada por el ejercicio es un estímulo endotelial crítico que se utiliza con frecuencia para evaluar la disfunción endotelial. El entrenamiento aeróbico regular en pacientes con IC sistólica, independiente de la causa, se asocia con restauración de la capacidad de vasodilatación dependiente del endotelio. La intensidad y la modalidad del ejercicio también son importantes de considerar. Mientras que los episodios agudos de ejercicio aislado pueden aumentar las citoquinas pro-inflamatorias y los marcadores de injuria endotelial, el ejercicio moderado y regular sí es efectivo para prevenir la remodelación del VI y detener, o incluso revertir, el proceso de daño endotelial.

\section{CONCLUSIÓN}

El endotelio tiene un papel crítico en una gran variedad de enfermedades, incluyendo la enfermedad vascular periférica, el accidente cerebrovascular, la diabetes, la insuficiencia renal crónica, el crecimiento tumoral, las enfermedades infecciosas y el centro de esta revisión, la IC. Debido a que la función endotelial es un marcador para el inicio y progresión de la IC, se justifica tener un enfoque más agresivo que permita la detección temprana y la optimización de la función endotelial anormal. $\mathrm{Si}$ bien, muchos agentes disponibles disminuyen la disfunción endotelial indirectamente en el corto plazo, más estudios de reversibilidad de largo plazo deben ser conducidos para demostrar los beneficios clínicos significativos en pacientes con IC.

\section{REFERENCIAS BIBLIOGRÁFICAS}

1. McMurray JJ, Adamopoulos S, Anker SD, Auricchio A, Böhm M, Dickstein K, Falk V, et al. ESC Guidelines for the diagnosis and treatment of acute and chronic heart failure 2012: The Task Force for the Diagnosis and Treatment of Acute and Chronic Heart Failure 2012 of the European Society of Cardiology. Developed in collaboration with the Heart Failure Association (HFA) of the ESC. Eur J Heart Fail. 2012 Aug;14(8):803-69. doi: 10.1093/eurjhf/hfs105.

2. Rajendran $P$, Rengarajan $T$, Thangavel J, Nishigaki Y, Sakthisekaran D, Sethi G, Nishigaki I. The vascular endothelium and human diseases. Int J Biol Sci. 2013 Nov 9;9(10):1057-69. doi: 10.7150/ijbs.7502. eCollection 2013. 
3. Durand MJ, Gutterman DD. Diversity in mechanisms of endothelium-dependent vasodilation in health and disease. Microcirculation. 2013;20(3):239-47. doi: 10.1111/micc. 12040.

4. Michiels C. Endothelial cell functions. J Cell Physiol. 2003 Sp;196(3):430-43.

5. van Hinsbergh VW. Endothelium-role in regulation of coagulation and inflammation. Sem Immunopathol. 2012 Jan;34(1):93-106. doi: 10.1007/s00281011-0285-5.

6. Shantsila E, Wrigley BJ, Blann AD, Gill PS, Lip GY. A contemporary view on endothelial function in heart failure. Eur J Heart Fail. 2012 Aug;14(8):873-81. doi: 10.1093/eurjhf/hfs066.

7. Lerman A, Zeiher AM. Endothelial function cardiac events. Circulation. 2005 Jan 25;111(3):363-8.

8. Christensen HM, Schou M, Goetze JP, Faber J, Frystyk J, Flyvbjerg A, Kistorp C. Body mass index in chronic heart failure: association with biomarkers of neurohormonal activation, inflammation and endothelial dysfunction. BMC cardiovasc Disord. 2013 Oct 1;13(1):80. doi: 10.1186/1471-2261-13-80.

9. Heusch G, Libby P, Gersh B, Yellon D, Böhm M, Lopaschuk G, Opie L. Cardiovascular remodelling in coronary artery disease and heart failure. Lancet. 2014 May 31;383(9932):1933-43. doi: 10.1016/ S0140-6736(14)60107-0.

10. Santini L, Capria A, Di Molfetta A, Mahfouz K, Panattoni G, Minni V, Sergi D, Forleo GB, Romeo F. Endothelial dysfunction is a marker of systemic response to the cardiac resynchronization therapy in heart failure. J Card Fail. 2013 Jun;19(6):419-25. doi: 10.1016/j.cardfail.2013.05.001.

11. Marti CN, Gheorghiade M, Kalogeropoulos AP, Georgiopoulou VV, Quyyumi AA, Butler J. Endothelial dysfunction, arterial stiffness, and heart failure. J Am Coll Cardiol. 2012 Oct 16;60(16):145569. doi: 10.1016/j.jacc.2011.11.082.

12. Dworakowski R, Walker S, Momin A, Desai J, ElGamel A, Wendler O, Kearney MT, Shah AM. Reduced nicotinamide adenine dinucleotide phosphate oxidase-derived superoxide and vascular endothelial dysfunction in human heart failure. J Am Coll Cardiol. 2008 Apr 8;51(14):1349-56. doi: 10.1016/j. jacc.2007.12.033.

13. Matsue $Y$, Suzuki M, Nagahori W, Ohno M, Matsumura A, Hashimoto Y, Yoshida K, Yoshida M Endothelial dysfunction measured by peripheral arterial tonometry predicts prognosis in patients with heart failure with preserved ejection fraction. J Am Coll Cardiol. 2013 Sept 20;59(13s1):36-40. doi: 10.1016/j.ijcard.2012.09.021.
14. Akiyama E, Sugiyama S, Matsuzawa Y, Konish M, Suzuki H, Nozaki T, Ohba K, Matsubara J, et al. Incremental prognostic significance of periphera endothelial dysfunction in patients with heart failure with normal left ventricular ejection fraction. J Am Coll Cardiol. 2012 Oct 30;60(18):1778-86. doi: 10.1016/j.jacc.2012.07.036.

15. Madamanchi NR, Runge MS. Redox signaling in cardiovascular health and disease. Free Radic Biol Med. 2013 Apr 11;61C:473-501. doi: 10.1016/j. freeradbiomed.2013.04.001

16. Hollenberg S.M, Klein LW, Parrillo JE, Scherer M, Burns D, Tamburro P, Oberoi M, Johnson MR, Costanzo MR. Coronary endothelial dysfunction after heart transplantation predicts allograft vasculopathy and cardiac death. Circulation. 2001 Dec 18;104(25):3091-6.

17. Adamopoulos S, Parissis JT, Kremastinos DT. Endothelial dysfunction in chronic heart failure: clinical and therapeutic implications. Eur J Intern Med. 2002 Jun;13(4):233-9.

18. Franciosa JA, Taylor AL, Cohn JN, Yancy CW, Ziesche S, Olukotun A, Ofili E, Ferdinand K, Loscalzo J, Worcel M; A-HeFT Investigators. African-American heart failure trial (A-HeFT): rationale, design, and methodology. J Card Fail. 2002 Jun;8(3):128-35.

19. Edvinsson ML, Uddman E, Edvinsson L, Andersson SE. Brain natriuretic peptide is a potent vasodilator in aged human microcirculation and shows a blunted response in heart failure patients. J Geriatr Cardiol. 2014 Mar;11(1):50-6. doi: 10.3969/j.issn.1671-5411 .2014 .01 .004

20. Solomon SD, Zile M, Pieske B, Voors A, Shah A, Kraigher-Krainer E, Shi V, Bransford T, Takeuchi $M$, et al., The angiotensin receptor neprilysin inhibitor LCZ696 in heart failure with preserved ejection fraction: a phase 2 double-blind randomised controlled trial. Lancet. 2012 Oct 20;380(9851):1387-95. doi: 10.1016/S0140-6736(12)61227-6.

21. Farquharson CA, Struthers AD. Spironolactone increases nitric oxide bioactivity, improves endothelial vasodilator dysfunction, and suppresses vascular angiotensin I/angiotensin II conversion in patients with chronic heart failure. Circulation. $2000 \mathrm{Feb}$ 15;101(6):594-7.

22. Strey CH, Young JM, Molyneux SL, George PM, Florkowski CM, Scott RS, Frampton CM. Endotheliumameliorating effects of statin therapy and coenzyme $Q<$ sub $>10</$ sub $>$ reductions in chronic heart failure. Atherosclerosis. 2005 Mar;179(1):201-6.

23. Hambrecht R, Hilbrich L, Erbs S, Gielen S, Fiehn E, Schoene N, Schuler G. Correction of endothelial dysfunction in chronic heart failure: additional effects of exercise training and oral L-arginine supplementation. J Am Coll Cardiol. 2000 Mar 1;35(3):706-13.

24. Rössig L, Hoffmann J, Hugel B, Mallat Z, Haase A, Freyssinet JM, Tedgui A, Aicher A, Zeiher AM, Dimmeler S. Vitamin C inhibits endothelial cell apoptosis in congestive heart failure. Circulation. 2001Oct 0;104(18):2182-7.

25. Teerlink JR, Cotter G, Davison BA, Felker GM, Filippatos G, Greenberg BH, Ponikowski P, Serelaxin, recombinant human relaxin-2, for treatment of acute heart failure (RELAX-AHF): a randomised, placebocontrolled trial. Lancet. 2013 Jan 5;381(9860):2939. doi: 10.1016/S0140-6736(12)61855-8.

26. Teichman SL, Unemori E, Teerlink JR, Cotter G, Metra M. Relaxin: review of biology and potential role in treating heart failure. Curr Heart Fail Rep. 2010 Jun;7(2):75-82. doi: 10.1007/s11897-010-0010-z.

27. Bruneau BG. The developmental genetics of congenital heart disease. Nature. 2008 Feb 21;451(7181):943-8. doi: 10.1038/nature06801.

28. Ferrari R, Rizzo P. The Notch pathway: a novel target for myocardial remodelling therapy? Eur Heart J. 2014 Jun 26; pii: ehu244.

29. Van Craenenbroeck EM, Conraads VM. Mending injured endothelium in chronic heart failure: a new target for exercise training. Int J Cardiol. 2013 Jun 20;166(2):310-4. doi: 10.1016/j. ijcard.2012.04.106.

\section{Conflictos de interés}

El Doctor Andrés F. Buitrago dicta conferencias para Abbott, Amarey, AstraZeneca, Bayer, Biopas, Biopisfar, Boehringer Ingelheim, Bristol-Myers \& Squibb, Biotoscana, Medtronic, Merck Serono, Merck Sharp \& Dohme, Novartis, Pacific Rubiales Pfizer, Sanofi. Es consultor para AstraZeneca, Boehringer Ingelheim, Bristol-Myers \& Squibb, Pacific Rubiales.

La Dra. Beatriz Wills declara no tener conflictos de interés.

\section{Correspondencia:}

Dra. Beatriz Wills

Correo electrónico: b.wills245@uniandes.edu.co 